\title{
$\mathbf{A}_{1}$ 腺苷受体的同源模建及其结构验证
}

\author{
柯艳蓉金宏威刘振明张亮仁* \\ (北京大学医学部药学院, 天然药物及仿生药物国家重点实验室, 北京 100191)
}

\begin{abstract}
摘要：采用同源模建的方法构建了 $\mathrm{A}_{1}$ 腺苷受体的三维结构, 并与拮抗剂分子 DPCPX 对接, 将得到的复合物结 构进行 $5 \mathrm{~ns}$ 的分子动力学模拟, 以最后 $2 \mathrm{~ns}$ 的平均结构和平衡后抽取的 11 帧构象共 12 个蛋白结构为研究对 象, 用包含 52 个活性分子和 1000 个诱饵分子的测试库, 分别通过 DOCK、VINA 和 GOLD 三种对接软件进行评 价, 最终得出合理的蛋白质模型. 根据 top $10 \%$ 的富集因子(EF)和 ROC 曲线下面积(AU-ROC)的计算结果, 我们认 为 GOLD 是最适合 $\mathrm{A}_{1}$ 腺苷受体的对接软件, 而 12 个蛋白质结构中 F5 和 Favg 的三维结构模型比较合理, 可以 作为进一步大规模虚拟笁选的模型.
\end{abstract}

关键词：分子动力学模拟； $\mathrm{A}_{1}$ 腺苷受体；同源模建； GOLD； 虚拟筛选 中图分类号: 0641

\section{Homology Modeling and Structure Validation of the Adenosine $\mathbf{A}_{1}$ Receptor}

\author{
KE Yan-Rong JIN Hong-Wei LIU Zhen-Ming ZHANG Liang-Ren * \\ (State Key Laboratory of Natural and Biomimetic Drugs, School of Pharmaceutical Sciences, Health Science Center, \\ Peking University, Beijing 100191, P. R. China)
}

\begin{abstract}
A three dimensional structure model of the adenosine $\mathrm{A}_{1}$ receptor was built using homology modeling. The antagonist DPCPX was docked into the model protein to form a receptor-ligand complex. A molecular dynamics simulation over $5 \mathrm{~ns}$ was performed for this complex. We selected 12 protein structures, including the average structure obtained from the last $2 \mathrm{~ns}$ of the simulation and 11 frames extracted after equilibration for the study. A database comprising 52 active antagonists and 1000 decoys was then docked into the 12 protein models using DOCK, VINA, and GOLD software packages and these molecules were ranked by their docking scores. The best model protein with the highest enrichment factor (EF) and the largest area under the ROC (AU-ROC) was chosen for further study. The results from the enrichment factor at $10 \%$ of the ranked database $\left(\mathrm{EF}_{10}\right)$ and AU-ROC calculations indicate that GOLD is the best virtual screening software for the adenosine $A_{1}$ receptor. GOLD docking results suggest that optimized adenosine $A_{1}$ receptor protein structures, Favg and F5, can be used for virtual screening and for novel design to discover more potent antagonists.
\end{abstract}

Key Words : Molecular dynamics simulation; Adenosine $A_{1}$ receptor; Homology modeling; GOLD; Virtual screening

腺苷受体是 $\mathrm{G}$ 蛋白偶合受体(GPCR)蛋白受体 家族成员, 其主要结构特征包括了七次跨膜的疏水 螺旋区(transmembrane, TMl-TM7)、N 端区、三个胞
外 LOOP 区(extracellular loop, EL1-EL3)、三个胞内 LOOP 区(intracellular loop, IL1-IL3)和 C 端区. 胞内 IL3 和 C 端区与 $\mathrm{G}$ 蛋白偶联, 从而影响环磷酸腺苷

Received: May 19, 2010; Revised: June 7, 2010; Published on Web: July 14, 2010.

*Corresponding author. Email: liangren@bjmu.edu.cn; Tel: +86-10-82802567.

The project was supported by the National S\&T Major Project Foundation, China (2009ZX09501-002).

国家科技重大专项关键技术基金(2009ZX09501-002)资助项目

C Editorial office of Acta Physico-Chimica Sinica 
酶或磷酸脂酶 $\mathrm{C}$ 的活性, 使细胞内产生第二信使, 并 通过信息传递, 参与细胞物质代谢的调节和基因转 录的调控. 腺苷受体有四种亚型, 分别为 $\mathrm{A}_{1} 、 \mathrm{~A}_{2 \mathrm{~A}}$ 、 $\mathrm{A}_{2 \mathrm{~B}} 、 \mathrm{~A}_{3}{ }^{[1]}$, 并且通过内源性的腺苷调节多种生理功 能. 腺苷受体有其独特的药理学特征、组织分布和偶 联蛋白, 作为药物靶标, 其应用领域相当广泛, 主要 可应用于局部缺血性疾病(大脑和心脏)、睡眠功能 障碍、免疫功能和炎症功能紊乱、癌症等的治疗. 目 前, 已经设计合成出一系列的腺苷受体激动剂和拮 抗剂, 并且有部分化合物已进入临床研究.

$A_{1}$ 受体主要作用于中枢神经系统和外周循环 系统, 并且对机体有促进免疫的作用. $\mathrm{A}_{1}$ 受体集中 分布在脑和脊髓中, 主要是在海马, 小脑, 上丘脑, 皮 质 I、IV、VI 中大量存在. 另外, $A_{1}$ 受体也广泛分布 于心脏, 在肾脏、肺和膀胱、脂肪等组织细胞中也有 表达. $A_{1}$ 受体作为潜在的药物靶标 ${ }^{[2-3]}$, 吸引了很多 的药物工作者对它的研究. 因为,一方面 $\mathrm{A}_{1}$ 受体的 拮抗剂主要应用于抗高血压药、钾离子保留的利尿 药、认知功能增强药、缓和阿尔兹海默综合症 ${ }^{[4-5]}$, 也 可用于治疗痴呆、缓解焦虑 ${ }^{6}$, 以及治疗充血性心率 衰竭患者的急性肾功能紊乱 ${ }^{[7-8]}$ 等; 另一方面, $\mathrm{A}_{1}$ 受 体拮抗剂的发展可作为蛋白受体药理学特征的分子 探针. 该受体拮抗剂分子设计主要以天然的咖啡因、 茶碱为母核结构进行化学修饰, 主要有两种类型: 黄嘌呤结构衍生物和非黄嘌呤结构衍生物(多聚杂 环衍生物). 目前, $\mathrm{A}_{1}$ 受体的晶体结构尚未解析出来, 所以通常采用同源模建的方法构建该受体的模型. 目前, 已报道 $\mathrm{A}_{1}$ 受体的模型, 主要是以牛视紫红质 受体为模板 ${ }^{[9-11]} .2007$ 年, $\beta_{2}$-肾上腺素受体晶体结构 被解析出来后 ${ }^{[12]}$, Yuzlenko 等 ${ }^{[13]}$ 分别以牛视紫红质 受体和 $\beta_{2}$-肾上腺素受体为模板进行 $\mathrm{A}_{1}$ 受体的同源 模建, 发现 $\beta_{2}$-肾上腺素受体更适合于腺苷受体的建 模. 2008 年, Jaakola 等 ${ }^{[14]}$ 解析了 $\mathrm{A}_{2 \mathrm{~A}}$ 受体的晶体结 构, 给腺苷受体家族其他亚型受体的模建提供了更 好的模板.

本文通过同源模建的方法模拟了 $\mathrm{A}_{1}$ 受体的三 维结构, 将得到的模型与 DPCPX 拮抗剂进行分子 对接, 得到受体-配体复合物结构. 将该复合物置于 磷脂双分子层中进行分子动力学模拟, 使受体模型 结构得到优化. 将包含 52 个 $\mathrm{A}_{1}$ 受体拮抗剂的测试 库分别与动力学平衡过程中抽取的 11 个构象和平 均结构进行对接, 以活性分子的 top $10 \%$ 的富集因子 (enrichment factor, $\mathrm{EF}$ )和ROC曲线下面积(AU-ROC)
大小作为结果的评价依据, 以便为进行大规模虚拟 篮选提供合理的受体结构.

\section{1 计算方法}

$\mathrm{A}_{2 \mathrm{~A}}$ 受体(分辨率 $0.26 \mathrm{~nm}, \mathrm{PDB}$ 编号 $3 \mathrm{EML}$ )结 构数据来源于 PDB 蛋白晶体结构数据库 ${ }^{[15]}$. $\mathrm{A}_{1}$ 受体 的氨基酸序列来源于 SWISS-PROT 数据库(登记号 为 P30542 $)^{[16]}$. 所有蛋白模建、模型优化、分子对接、 动力学模拟计算均在 DELL PowerEdge 2950(Xeon $\mathrm{E} 5410-2.33 \mathrm{G} \times 2$, 内存 $8.0 \mathrm{G})$ 计算机工作站完成, 所用 程序为 Sybyl 6.9(Tripos 公司)和 Discovery Studio (DS) 2.1(Accelrys 公司)分子设计软件包 ${ }^{[17-18]}$, 计算中 选用的各项参数除特别说明外均使用缺省值.

\section{1 同源模建}

$\mathrm{A}_{1}$ 受体由 326 个氨基酸残基组成. 序列比对采 用 DS 2.1 中的 Align123 程序, 并根据 A 型 G 蛋白 偶联受体家族中高度保守的氨基酸残基位置对结果 进行手动调整. 采用 DS 2.1 中的 MODELER 程序 对 $\mathrm{A}_{1}$ 受体模型进行搭建, 使用 BLOSUM 矩阵, 多 序列断点罚分 (gap open penalty) 为 5.0, 多序列断点 伸展罚分 (gap extension penalty)为 0.05 . 由程序自动 生成 10 个模型, 选取概率密度函数 (probability density functions, PDF)对蛋白质几何性质打分最高的 模型, 用 Procheck 程序进行合理性评价.

\section{2 分子对接}

首先使用 SYBYL 6.9 软件对小分子和蛋白进 行处理. $A_{1}$ 受体拮抗剂 DPCPX 分子的初始结构使 用 sketch 模块构建, 如图 1 所示, 并对小分子加氢 和加 Gasteige -Hückel 电荷. 对所构建的初始结构依 次进行 1000 步最陡下降法和 1000 步共轭梯度法优 化. 使用 Biopolymer 模块对蛋白模型加极性氢和赋 予 AMBER7 FF99 电荷. 分子对接使用 GOLD 3.0.1 程序 ${ }^{[19]}$, 将 DPCPX 分子置人受体的活性位点. 我们 根据文献报道的突变数据 ${ }^{[20-21]}$ 来定义 $\mathrm{A}_{1}$ 受体的活 性位点, 即以 Ser94 和 His278 两个关键残基的中心

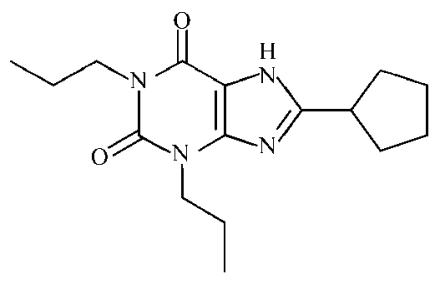

图 1 DPCPX 化学结构

Fig.1 Chemical structure of DPCPX 
坐标为中心, 半径为 $2.5 \mathrm{~nm}$ 的球形空间为活性口 袋. 该口袋包含的关键残基有 Leu88、Thr91、Gln92、 Ser94、His251 和 His278 ${ }^{[22]}$. 采用遗传算法 $(\mathrm{GA})$ 进行 蛋白-配体对接, 对接时考虑配体柔性和蛋白部分柔 性. 采用 Goldscore 打分函数, 选择打分值最高的小 分子结合构象进行动力学研究.

\section{3 分子动力学模拟}

对于 DPCPX 分子使用 Gaussian 03 程序 ${ }^{[23]}$, 用 $\mathrm{HF}$ 方法, 在 $6-31 \mathrm{G}^{*}$ 基组水平下计算该分子的静电 势. 然后用 AMBER 8.0 软件包中的 Antechamber 程 序, 用 RESP 方法计算 DPCPX 的部分原子电荷 ${ }^{[24]}$, 得到小分子力场文件. MD 模拟使用 Gromacs 3.2.1 程序包 ${ }^{[25-26]}$, 用 ' genbox'命令将对接得到的复合物 置人 DPPC 256 膜中. 膜中心 56 个磷脂分子被自动 删除避免与蛋白产生碰撞. 然后, 对整个体系添加 $\mathrm{SPC}$ 水模型, 添加 13 个氯离子, 使体系保持电中性. 模拟体系共有 58147 个原子, 其中 3078 个蛋白质 原子, 200 个磷脂膜分子, 15011 个水分子, 13 个氯 离子.

采用 Gromacs $96^{[27]}$ 力场, 将蛋白质复合物-膜水体系分别用最陡下降法和共轭梯度各进行 1000 步的能量优化, 以避免模拟时原子间的不合理碰撞; 然后, 束缚蛋白和小分子, 对膜-水体系进行 $200 \mathrm{ps}$ 的动力学模拟; 再束缚蛋白主链和小分子, 对蛋白 侧链-膜-水体系进行 $200 \mathrm{ps}$ 的动力学模拟; 最后对 整个体系进行 $5 \mathrm{~ns}$ 的恒温恒压分子动力学模拟 计算. 动力学过程中, 步长设为 $2 \mathrm{fs}$; 使用 Berendsen 等 ${ }^{[28]}$ 提出的温度和压力耦合方法, 使系统温度保持 在 $310 \mathrm{~K}$, 耦合时间 $0.1 \mathrm{ps}$; 压力保持在 $105 \mathrm{~Pa}$, 采用 $0.5 \mathrm{ps}$ 耦合时间模拟自由水. 模拟水分子的等温压 缩系数设为 $4.5 \times 10^{-10} \mathrm{~Pa}^{-1}$, 用 LINCS (linear constraint)算法 ${ }^{[29}$ 约束所有原子的键长, 计算距离小于 1 $\mathrm{nm}$ 的带电基团之间的静电相互作用; 用 PME(particle-mesh Ewald)方法 ${ }^{[30}$ 计算长程静电相互作用, 格 点宽度设为 $0.12 \mathrm{~nm}$; Lennard-Jones 相互作用的截 断距离设为 $1 \mathrm{~nm}$.

在最后 $2 \mathrm{~ns}$ 模拟时间内, 每隔 20 帧保留一个 构象, 总共抽取了 11 个构象, 分别命名为 Fl-Fll. 再计算得到最后 $2 \mathrm{~ns}$ 模拟时间内的平均结构, 并对 该结构进行真空中的 800 步最陡下降法和 1200 步 的共轭梯度法的能量优化, 获得最终结构命名为 Favg. 这 12 个 DPCPX-A A $_{1}$ 受体的复合物结构将用于 进一步模型评价.

\section{4 小分子数据库的制作}

从 MDDR(Elevier MDL, San Leandro CA)数据 库中, 随机抽取 52 个 $\mathrm{A}_{1}$ 受体的拮抗剂, 与来自薛定 谔网站上的 1000 个分子量为 400 的 decoys, 组成一 个含有 1052 个分子的测试库. 使用 Concord v 6.1.3 程序得到测试库分子的三维结构用于虚拟篮选评 价.

\section{5 模型评价}

虚拟篮选评价采用 VINA、DOCK 4.0、GOLD 3.0.1 三种对接软件. 使用 DOCK 4.0 软件包 ${ }^{[31-32]}$ 进 行对接时, 通过 Sphgen 程序对蛋白负像进行聚类, 以每个复合物结构中的配体分子定义活性位点, 格 点球半径为 $1 \mathrm{~nm}$. 对接结果以能量打分进行排序. 使用 VINA 软件 ${ }^{[33]}$ 进行对接, 受体和小分子先后在 MGLTools 1.4.6 中打开进行对接前文件处理, 对于 受体结构, 添加极性氢原子, 计算电荷. 用小分子定 义活性位点的中心, 并界定 $2 \mathrm{~nm} \times 2 \mathrm{~nm} \times 2 \mathrm{~nm}$ 的对 接格点范围. 对接结果进行能量排序. 使用 GOLD 3.0.1 软件 ${ }^{[19]}$ 进行对接, 以配体小分子定义活性位点, 以小分子为中心半径为 $2.5 \mathrm{~nm}$ 的球形空间为活性 口袋. 采用遗传算法(GA)进行蛋白-配体对接, 对接 时考虑配体柔性和蛋白部分柔性. 采用 Goldscore 打分作为打分函数, 以 GOLD fitness function 值进 行排序.

\section{2 结果与讨论}

\section{1 同源模型的评价}

通过 Align123 程序的序列比对如图 2 所示, 结 果表明 $\mathrm{A}_{1}$ 和 $\mathrm{A}_{2 \mathrm{~A}}$ 具有较高的序列相似度(similarity: $71.6 \%$ ) 和一致性(identity: $54.1 \%$ ), 是以其他 GPCRs 蛋白晶体结构为模板的多序列比对达不到 的. 而且, 模建模型中保留了腺苷受体 TM3 和 EL2 二硫键 $\left(\mathrm{A}_{1}\right.$ : Cys80-Cys169)的特征, 且EL3中Cys260Cys263二硫键特征(见图3)与 $\mathrm{A}_{2 \mathrm{~A}}$ 晶体结构二硫键特 征相符 ${ }^{[14]}$. 我们使用 Procheck 程序对模型结构的立 体化学参数进行了评价, 该程序可以凭经验比较给 定蛋白质结构与最合理的蛋白质结构之间立体化学 性质的差异. 评价结果中主要考察所有氨基酸残基骨 架的二面角分布, 并通过程序生成的Ramachandran 图来表示(见图4). 我们可以看到模建的蛋白质有 93.5\%的残基落人最佳区域(the most favoured regions), $5.8 \%$ 的残基落人其它许可区 (additional allowed regions), $0.7 \%$ 的残基落人勉强许可区(gener- 


P30542|AA1R_HUMAN
3EML

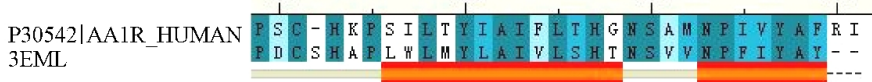

图 $2 \mathbf{A}_{1}$ 腺苷受体与 $\mathbf{A}_{2 \mathrm{~A}}$ 腺苷受体序列比对图

Fig.2 Sequence alignment of adenosine $A_{1}$ receptor and adenosine $A_{2 A}$ receptor

TM1 to TM7 indicate the seven transmembrane domains of G-protein coupled receptors (GPCRs), P30542|AAlR_HUMAN and 3EML refer to the amino acid sequences of adenosine $\mathrm{A}_{1}$ receptor and adenosine $\mathrm{A}_{2 \mathrm{~A}}$ receptor, respectively. (identity, $54.1 \%$; similarity, $71.6 \%$ )

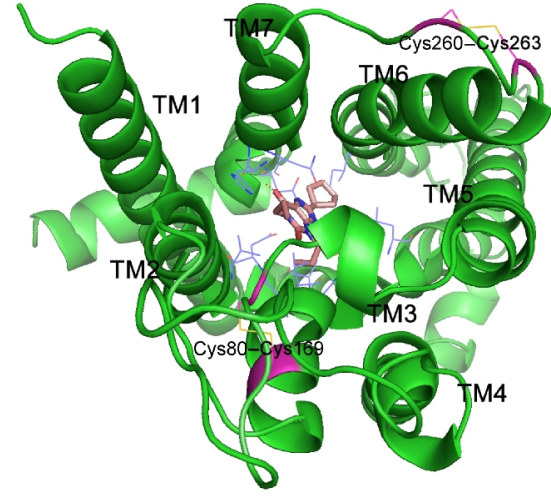

图 $3 \mathbf{A}_{1}$ 受体同源模建后的模型

Fig.3 Adenosine $A_{1}$ receptor structure constructed by homoloy modeling

Cys80-Cys169 between TM3 and EL2; Cys260-Cys263 in EL3

ously allowed regions), 无残基落人不允许区(disallowed regions). 上述结果说明模建得到的 $\mathrm{A}_{1}$ 受体模 型氨基酸二面角结构是比较合理的.

\section{2 受体抑制状态模型的构建和分子动力学模拟}

DPCPX 是黄嘌呤结构的高选择 $\mathrm{A}_{1}$ 受体的拮抗 剂分子, 该分子与 $\mathrm{A}_{1}$ 受体的亲和力 $K_{i}$ 值为 3.9 $\mathrm{nmol} \cdot \mathrm{L}^{-1}$, 其选择性表现为该分子与其它亚型受体 的亲和力 $K_{\mathrm{i}}$ 值分别为 $\mathrm{A}_{2 \mathrm{~A}}: 129 \mathrm{nmol} \cdot \mathrm{L}^{-1} 、 \mathrm{~A}_{2 \mathrm{~B}}: 56$ $\mathrm{nmol} \cdot \mathrm{L}^{-1} 、 \mathrm{~A}_{3}: 3980 \mathrm{nmol} \cdot \mathrm{L}^{-1[34]}$. 而且, 同位素 $\left[{ }^{3} \mathrm{H}\right]$ DPCPX 被广泛应用于 $A_{1}$ 和 $A_{2 B}$ 受体放射性结合实 验和突变实验研究. 因此, 我们选择 DPCPX 分子与 $A_{1}$ 模型进行分子对接. 选择 Goldscore 打分最好的 构象, 进行下一步的动力学模拟.

将 $\mathrm{A}_{1}$ 受体-DPCPX 复合物模型置于 DPPC 膜

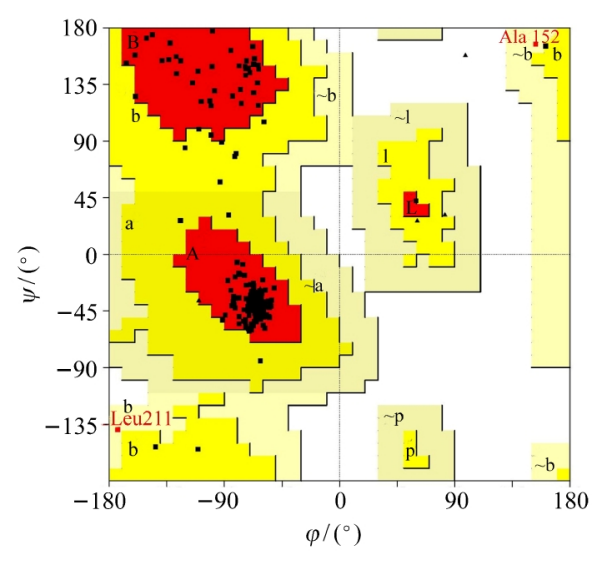

图 $4 \mathbf{A}_{1}$ 受体模型的 Ramachandran 图

Fig.4 Ramachandran plot of the modeled adenosine $A_{1}$ receptor

$\mathrm{A}, \mathrm{B}$, and $\mathrm{L}$ represent the residues in most favored regions; $\mathrm{a}, \mathrm{b}, \mathrm{l}$, and $\mathrm{p}$ represent the residues in additional allowed regions; $\sim \mathrm{a}, \sim \mathrm{b}, \sim 1$, and $\sim$ p represent the residues in generously allowed regions.

中进行了 $5 \mathrm{~ns}$ 的分子动力学模拟(图 5). 在模拟过 程中, DPCPX 小分子拮抗剂诱导大分子受体构象逐 渐变化, 最终达到能量平衡. 相对于起始结构的均方 根偏差(RMSD)计算结果证明了轨迹的稳定性. 图 6 给出了 $5 \mathrm{~ns}$ 模拟时间内蛋白质骨架和小分子的 RMSD 值随时间的变化曲线. 可以看到, 在开始的 $1.5 \mathrm{~ns}$ 模拟时间内, 蛋白质骨架的 RMSD 值(黑色曲 线)缓慢上升达到 $0.25 \mathrm{~nm}$, 表明了蛋白分子的运动 和结构的不断变化. 而在 $1.5 \mathrm{~ns}$ 之后, RMSD 值趋于 稳定, 在 $0.25-0.3 \mathrm{~nm}$ 之间上下波动. 小分子的 RMSD 值(红色曲线)变化不大, 一直稳定在 $0.1 \mathrm{~nm}$ 范围内, 处于平衡的动力学状态. 从该体系在模拟过 程中的势能变化曲线(图 7)可以看出, 体系的势能在 


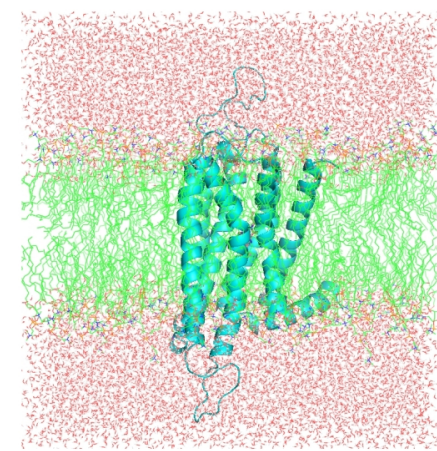

图 5 动力学模拟过程中 DPPC256 磷脂膜- $\mathrm{A}_{1}$ 受体DPCPX 体系的初始结构模型

Fig.5 Initial model containing DPPC256, $\mathrm{A}_{1}$ receptor, and DPCPX for the molecular dynamics simulation

最初的十几 ps 时间内迅速降低, 然后随着模拟的进 程势能逐渐降低, 并从 $3 \mathrm{~ns}$ 开始势能趋于稳定直到 $5 \mathrm{~ns}$, 说明该体系已经达到平衡. 根据分子动力学模 拟结果, 我们从 $3 \mathrm{~ns}$ 起每隔 20 帧取一个构象, 共取 得 11 个构象 (Fl-Fl1). 再加上 3-5 ns 时间内的平均 结构(Favg), 共得到 12 个结构.

图 8 给出了 Favg 中, 小分子和蛋白的结合模 式. 我们可以看到, DPCPX 分子主要结合区域是 TM2、TM3、TM6、TM7，残基 His278、Val58、Val87 和残基 Leu90、Thr91、Ala54、Asp55 分别构成了两 个疏水性口袋, 与 DPCPX 分子的 1 位和 3 位的丙 基有疏水作用; Leu51、Leu98、Asn284 和 Leu239 残 基也构成了疏水性口袋, 与 8 位的环戊烷有疏水作 用. 此外, DPCPX 的 4 位羰基与 Asn280 和 Ser281 肽键上的氨基形成氢键 (氢键键长: $0.2 \mathrm{~nm}$ ), 7 位氨

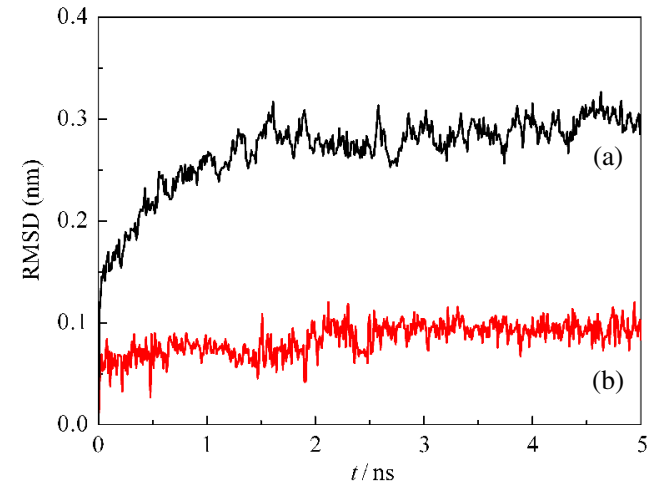

图 $65 \mathrm{~ns}$ 动力学模拟过程中蛋白质骨架(a)和小分子拮抗 剂 DPCPX(b)的均方根偏差(RMSD)与时间的关系曲线

Fig.6 Root mean square deviation (RMSD) $v$ s time of the atoms from their initial positions during the $5 \mathrm{~ns}$ simulation of protein backbone (a) and DPCPX (b)

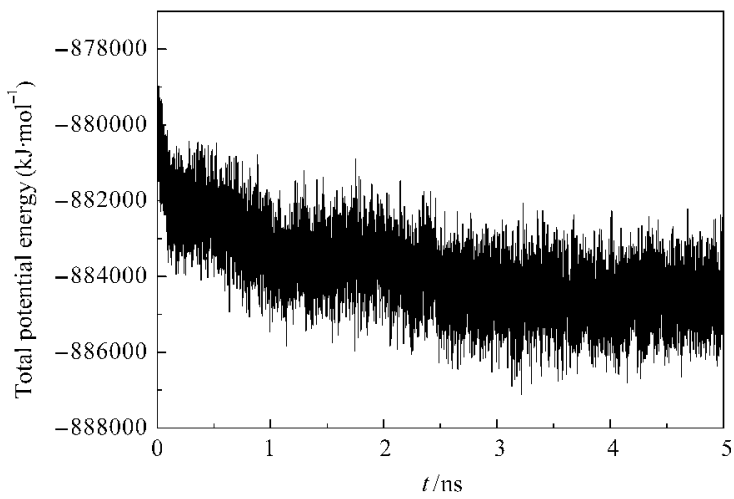

图 7 动力学模拟过程中体系的势能变化

Fig.7 Total potential energy changes of the system during molecular dynamics simulation

基氮与 Ser94 的差基氧具有氢键相互作用(氢键键 长: $0.3 \mathrm{~nm}$ ). 根据文献报道, 对于 $\mathrm{A}_{1}$ 腺苷受体, 突变 Ser94 和 His 278 这两个氨基酸残基, 则蛋白完全丧 失与拮抗剂的结合. 我们的对接结果与突变实验结 果基本符合 ${ }^{[20-22]}$.

\section{3 用测试数据库验证模型}

采用 DOCK 4.0、VINA 和 GOLD 3.0.1 对接软 件, 将分子动力学模拟得到的 12 个蛋白质模型与测 试库分子进行对接. 用 $\mathrm{EF}$ 和 AU-ROC 两个参数来 评价蛋白模型是否合理.

$\mathrm{EF}$ 是评价虚拟篮选好坏的一个重要指标, 它是 指, 在选择的排名前百分之几(例如 $10 \%$ )的数据集 中活性分子的浓度与初始活性分子浓度的比值. 这 个指标用于评价篮选中篮选出活性分子的阳性率. 富集因子计算公式为 ${ }^{[35]}$ :

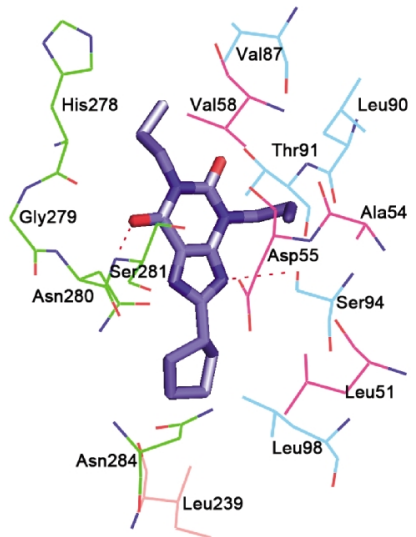

图 $8 \mathrm{DPCPX}$ 与 $\mathrm{A}_{1}$ 受体结合模式

Fig.8 Binding profile of DPCPX and adenosine $\mathrm{A}_{1}$ receptor

red: the residues in TM2; blue: the residues in TM3; orange: the residues in TM5; green: the residues in TM6 


$$
\mathrm{EF}_{\text {subset }}=\frac{\text { ligands }_{\text {selected }} / N_{\text {subset }}}{\text { ligands }_{\text {total }} / N_{\text {total }}}
$$

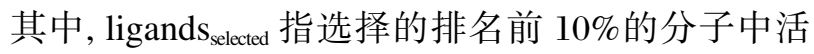
性小分子的数量, $N_{\text {subset }}$ 指选择的前 $10 \%$ 的小分子的

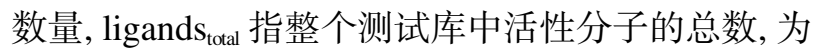
$52, N_{\text {total }}$ 指整个测试库分子的总数, 为 1052 .

ROC (receiver operator characteristic) 曲线描绘 的是敏感度 (sensitivity, Se) 和特异性(specificity, Sp) 的关系. AU-ROC 值指的是 ROC 曲线下的面积 ${ }^{[36-37]}$. 这个指标用于测试虚拟篮选策略的优劣, 当被评价 的篎选策略都篎选出相同比例的诱饵分子时, 篮出 活性分子的比例越高说明该策略对活性分子的敏感 度越高. AU-ROC 值是以具体数值表示 $\mathrm{ROC}$ 曲线 下的面积, 该值的区间是 $[0,1], 1$ 表示篮出 0 个诱饵 分子时篮出所有的活性分子; 0 表示篮出全部的诱 饵分子中不含有活性分子.

因此, 我们希望真正有活性的化合物能够尽可 能富集在前百分之十比例(top $10 \%$ )的篮选结果中, 我们也希望 AUC 曲线下面积能越大越好, 这样有 利于评价这 12 个蛋白结构的优劣性, 选择更合理的 蛋白结构和更合适的对接软件用于进一步大规模虚 拟篮选研究和小分子结合模式的研究. 将 12 个蛋白 质结构分别通过 DOCK 4.0、VINA、GOLD 3.0.1 三 个对接软件分别与测试数据库对接后, 对接结果用 相应的评分函数打分并进行排序和计算, 得到相关 的测试库中 52 个活性分子的 $\mathrm{EF}$ 和 AU-ROC 值(表

表 1 DOCK、GOLD 和 VINA 软件的对接评价结果 Table 1 Evaluations of docking results obtained by DOCK, GOLD, and VINA softwares

\begin{tabular}{|c|c|c|c|c|c|c|}
\hline \multirow{2}{*}{ Conformation } & \multicolumn{2}{|c|}{ DOCK } & \multicolumn{2}{|c|}{ GOLD } & \multicolumn{2}{|c|}{ VINA } \\
\hline & $\mathrm{EF}_{10}$ & $\overline{\text { AU-ROC }}$ & $\mathrm{EF}_{10}$ & $\overline{\text { AU-ROC }}$ & $\mathrm{EF}_{10}$ & AU-ROC \\
\hline F1 & 1.821 & 0.108 & 3.642 & 0.277 & 1.012 & 0.061 \\
\hline $\mathrm{F} 2$ & 0.607 & 0.049 & 3.035 & 0.249 & 2.225 & 0.173 \\
\hline F3 & 1.821 & 0.117 & 3.237 & 0.239 & 1.821 & 0.174 \\
\hline F4 & 1.821 & 0.128 & 4.046 & 0.284 & 1.416 & 0.117 \\
\hline F5 & 0.607 & 0.089 & 4.046 & 0.360 & 1.012 & 0.102 \\
\hline F6 & 1.214 & 0.128 & 2.630 & 0.217 & 2.023 & 0.144 \\
\hline F7 & 0.607 & 0.074 & 2.225 & 0.245 & 1.012 & 0.105 \\
\hline F8 & 1.821 & 0.163 & 2.225 & 0.227 & 1.012 & 0.072 \\
\hline F9 & 1.618 & 0.122 & 3.642 & 0.309 & 1.618 & 0.166 \\
\hline F10 & 0.607 & 0.068 & 3.439 & 0.293 & 1.821 & 0.165 \\
\hline F11 & 1.618 & 0.127 & 3.844 & 0.274 & 1.012 & 0.094 \\
\hline Favg & 1.012 & 0.102 & 4.046 & 0.346 & 2.023 & 0.122 \\
\hline Avg & 1.265 & 0.106 & 3.338 & 0.277 & 1.501 & 0.125 \\
\hline
\end{tabular}

$\mathrm{EF}_{10}$ : enrichment factor at $10 \%$ of the ranked database; AU-ROC: area under the ROC curve
表 2 DOCK、GOLD 和 VINA 软件配对 $t$ 检验的结果

Table 2 Results of DOCK, GOLD, and VINA softwares' paired samples test

\begin{tabular}{|c|c|c|c|c|c|}
\hline Software & onformation & Mean & Standard deviation & $t$ & $p$-value \\
\hline GOLD & Favg-F8 & 2.72356 & 5.23021 & 3.755 & $<0.001$ \\
\hline VINA & Fl-F2 & 0.39038 & 0.49240 & 5.717 & $<0.001$ \\
\hline DOCK & F8-F2 & 3.08731 & 2.62057 & 8.495 & $<0.001$ \\
\hline
\end{tabular}

1). 从表 1 中 $\mathrm{EF}_{10}$ 和 $\mathrm{AU}-\mathrm{ROC}$ 的均值, 可看出 DOCK、GOLD、VINA 三种对接软件中, 最适合 $\mathrm{A}_{1}$ 受体进行虚拟筛选的对接软件是 GOLD. 而且, 三种 对接软件都可以特异性地选择某些构象的蛋白结 构. 对于 DOCK 软件更趋向于选择 F8、F3、F4 蛋白 结构, VINA 软件更趋向于选择 F2、Favg、F6 蛋白结 构, 而 GOLD 软件更趋向于选择 F5、Favg、F4 蛋白 结构.

分别以三个软件中各自评价最高和评价最低的 两组蛋白模型对接计算中 52 个活性分子的对接能 量值作为样本数据, 利用 SPSS 软件进行了配对 $t$ 检 验统计学分析, 在 5\%显著性水平的条件下, 自由度 为 50 的 $t$ 临界值为 3.261. 选择的蛋白模型包括 Favg 和 F8(GOLD)、F2 和 F1(VINA) 以及 F8 和 F2 (DOCK), 分析结果见表 2. 可以看到, 对于 $\operatorname{GOLD}(t=$ $3.775, p<0.001) 、 \operatorname{VINA}(t=5.717, p<0.001)$ 和 DOCK $(t=8.495, p<0.001)$ 计算所得的 $t$ 值明显大于临界值, 说明对接中各自的两组蛋白的小分子对接能量存在 着显著性差异, 即 Favg 蛋白的虚拟篮选能力比 F8 蛋白强(GOLD); F1 蛋白的虚拟篮选能力比 F2 蛋白 强(VINA); F8 蛋白的虚拟篮选能力比 F2 蛋白强 (DOCK). 总之, DOCK、GOLD 和 VINA 对接软件可 以特异性地选择某些构象的蛋白质结构, 并可以区 分出蛋白模型的优劣.

应用 GOLD 软件进行虚拟篮选时, F4、F5 和 Favg 的 $\mathrm{EF}_{10}$ 值均为 4.046, 说明它们是 12 个蛋白质 模型中比较合理的结构. 此外, 这三个蛋白质结构的 曲线下面积 $(A U-R O C)$ 分别为 $0.284 、 0.360 、 0.346$, 说明 F5 和 Favg 是两个不错的模型, 可用于进一步 的大规模虚拟篮选和小分子拮抗剂结合模式的研 究.

\section{3 结 论}

用同源模建的方法对 $\mathrm{A}_{1}$ 受体的三维结构进行 构建, 经过磷脂双分子膜中的分子动力学模拟, 抽取 了动力学平衡中最后 $2 \mathrm{~ns}$ 的 11 个构象 $(\mathrm{Fl}-\mathrm{F} 11)$ 和 
计算动力学平衡中的平均构象 (Favg). 用包含 52 个 活性分子的测试库分别用 DOCK、VINA、GOLD 三 种软件与 12 个模型进行对接以验证模型结构, 从 $\mathrm{EF}_{10}$ 和 $\mathrm{AU}-\mathrm{ROC}$ 平均值结果分析表明 GOLD 软件 是这三个软件中最适合 $\mathrm{A}_{1}$ 受体虚拟篮选. 而且, 这 三种软件都具有篮选出最优构象模型的能力, 最优 构象与最劣构象的小分子对接能量存在着显著性差 异. 从 GOLD 软件对 12 个蛋白模型的选择中, 我们 可知 F5 和 Favg 是最合理的蛋白结构模型, 可进一 步用于大规模虚拟篮选和小分子拮抗剂结合模式的 研究.

\section{References}

1 Ralevic, V.; Burnstock, G. Pharmacol. Rev., 1998, 50: 413

2 Müller, C. E.; Stein, B. Curr. Pharm. Design, 1996, 2: 501

3 Poulsen, S. A.; Quinn, R. J. Bioorg. Med. Chem., 1998, 6: 619

4 Müller, C. E. Expert. Opin. Ther. Pat., 1997, 7: 419

5 Hess, S. Expert. Opin. Ther. Pat., 2001, 11: 1533

6 Maemoto, T. J. Pharmacol. Sci., 2004, 96: 42

7 Wilcox, C. S.; Welch, W. J.; Schreiner, G. F.; Belardinelli, L. J. Am. Soc. Nephrol., 1999, 10: 714

8 Gottlieb, S. S. Circulation, 2002, 105: 1348

9 Giordanetto, F.; Fossa, P.; Menozzi, G.; Schenone, S.; Bondavalli, F.; Ranise, A.; Mosti, L. J. Comput. Aided Mol. Des., 2003, 17: 39

10 Gutiérrez-de-terán, H.; Centeno, N. B.; Pastor, M.; Sanz, F. Proteins, 2004, 54: 705

11 Tuccinardi, T.; Ortore, G.; Manera, C.; Saccomanni, G.; Martinelli, M. Euro. J. Med. Chem., 2006, 41: 321

12 Cherezov, V. Science, 2007, 318: 1258

13 Yuzlenko, O.; Kiec-Kononowicz, K. J. Comput. Chem., 2009, 30: 14

14 Jaakola, V. P. Science, 2008, 322: 1211

15 http://www.gpcr.org

16 http://expasy.org/sprot/
17 SYBYL software. Version 6.9. St. Louis: Tripos. Associates. Inc.

18 Discovery Studio software. Version 2.1. San Diego: Accelrys. Inc.

19 Jones, G.; Willett, P.; Glen, R. C.; Leach, A. R.; Taylor, R. J. Mol. Biol., 1997, 267: 727

20 Barbhaiya, H.; McClain, R.; Ijzerman, A.; Rivkees, S. Mol. Pharmacol., 1996, 50: 1635

21 Olah, M. E.; Ren, H.; Ostrowski, J.; Jacobson, K. A.; Stiles, G. L. J. Biol. Chem., 1992, 267: 10764

22 Martinelli, A.; Tuccinardi, T. Mol. Res. Rev., 2008, 28: 247

23 Frisch, M. J.; Trucks, G. W.; Schlegel, H. B.; et al. Gaussian 03. Revision B.03. Wallingford, CT: Gaussian Inc., 2003

24 Bayly, C. I.; Cieplak, P.; Cornell, W. D.; Kollman, P. A. J. Phys. Chem., 1993, 97: 10269

25 Berendsen, H. J. C.; van der Spoel, D.; van Drunen, R. Comp. Phys. Commun., 1995, 91: 43

26 Lindahl, E.; Hess, B.; van der Spoel, D. J. Mol. Model., 2001, 7: 306

27 van der Spoel, D.; Buuren, A. R. V.; Tieleman, D. P.; Berendsen, H. J. J. Biomol. NMR, 1996, 8: 229

28 Berendsen, H. J. C.; Postma, J. P. M.; van Gunsteren, W. F.; Dinola, A.; Haak, J. R. J. Chem. Phys., 1984, 81: 3684

29 Hess, B.; Bekker, H.; Berendsen, H. J. C.; Fraaije, J. G. E. M. J. Comput. Chem., 1997, 18: 463

30 Darden, T.; York, D.; Pedersen, L. J. Chem. Phys., 1993, 98: 10089

31 Kuntz, I. D.; Blaney, J. M.; Oatley, S. J.; Langridge, R.; Ferrin, T. E. J. Mol. Biol., 1982, 161: 269

32 Ewing, T. J. A.; Kuntz, I. D. J. Comput. Chem., 1997, 18: 1175

33 Trott, O.; Arthur, J. O. J. Comput. Chem., 2010, 31: 455

34 Moro, S.; Gao, Z. G.; Jacobson, K. A.; Spalluto, G. Med. Res. Rev., 2006, 26: 131

35 Wei, B. Q.; Baase, W. A.; Weaver, L. H.; Mattews, B. W.; Shoichet, B. K. J. Mol. Biol., 2002, 322: 339

36 Hevener, K. E.; Zhao, W.; Ball, D. M.; Babaoglu, K.; Qi, J. J.; White, S. W.; Lee, R. E. J. Chem. Inf. Model., 2009, 49: 444

37 Truchon, J. F.; Bayly, C. I. J. Chem. Inf. Model., 2007, 47: 488 